\title{
Trans-Sacral Epiduroscopic-Assisted 1,414-nm Nd:YAG Laser Decompression for Lumbar Discal Cyst: A Report of 9 Cases
}

\author{
Farid Yudoyono ${ }^{1}$, Gyu Yeol $\mathrm{Ji}^{2}$, Sang Won Lee ${ }^{3}$, Jongsun Lee, Dong Ah Shin ${ }^{5}$ \\ ${ }^{1}$ Department of Neurosurgery, Hasan Sadikin Hospital, Padjadjaran University, College of Medicine, Bandung, Jawa Barat, Indonesia \\ ${ }^{2}$ Department of Neurosurgery, Guro Cham teun Teun Hospital, Seoul, \\ ${ }^{3}$ Department of Neurosurgery, Yonsei Barun Hospital, Seoul, \\ ${ }^{4}$ Department of Neurosurgery, Sewori Spine and Joint Hospital, Daejeon, \\ ${ }^{5}$ Department of Neurosurgery, Spine and Spinal Cord Institute, Severance Hospital, Yonsei University College of Medicine, Seoul, Korea
}

Corresponding Author: Dong Ah Shin, MD, PhD Department of Neurosurgery, Spine and Spinal Cord Institute, Severance Hospital, Yonsei University College of Medicine, 50-1, Yonsei-ro, Seodaemun-gu, Seoul 03722, Korea

Tel: $+82-2-2228-2150$ Fax: +82-2-393-9979

E-mail: shindongah@me.com

Received: January 25, 2018 Revised: March 4, 2018 Accepted: March 6, 2018

Prevalence of lumbar discal cyst is very low, it can cause low back pain and radiating leg pain when present. Currently, trans-sacral epiduroscopic-assisted, 1,414-nm Nd:YAG laser decompression (SELD) is commonly used for spinal pathologies. However, the use of the laser for spinal procedures can be limited due to the risk of thermal injury. We reviewed nine consecutive patients who underwent SELD ablation for discal cyst between 2014 and 2015. Each patient underwent diagnostic imaging, including simple radiographs, computed tomography with discography, and magnetic resonance imaging (MRI). Pain relief and clinical outcome assessment of patient satisfaction was the primary outcome measure. All patients presented with back pain and unilateral radiating pain. The discal cyst was located in the lumbar region in all patients. Preoperative MRI showed a connection between the cyst and the involved intervertebral disc. All patients obtained immediate relief of symptoms after the discal cyst was treated with a SELD-assisted, 1,414-nm Nd:YAG laser. The mean visual analogue scale (VAS) for back pain was $7.89 \pm 0.78$ preoperatively, $1.67 \pm 1.50$ at the 1 -month follow up, and $0.38 \pm 0.5$ at the final follow up ( $p<$ 0.01). All patients obtained excellent or good outcomes according to the modified MacNab's criteria. There were no complications. These cases demonstrated that trans-sacral, epiduroscopic-assisted, 1,414$\mathrm{nm} \mathrm{Nd:YAG} \mathrm{laser} \mathrm{decompression} \mathrm{was} \mathrm{a} \mathrm{safe,} \mathrm{viable,} \mathrm{and} \mathrm{efficacious} \mathrm{option} \mathrm{for} \mathrm{treating} \mathrm{lumbar} \mathrm{discal}$ cyst because it lowers the risk of muscle injury and can be performed under local anesthesia.

Key Words: Discal cyst, Low back pain, Magnetic ressonance imaging, Trans-sacral epiduroscopic assisted laser decompression

\section{INTRODUCTION}

Discal cysts are a rare cause of low back and radiating pain, and they usually occur in relatively young individuals. Discal cysts are characterized by distinct interface with their corresponding intervertebral disc ${ }^{5,6,15,19)}$. Although conventional surgery is the gold standard for treatment of discal cysts, it can lead to complications, such as tissue disruption, bleeding, and fibrosis. Therefore, SELD Currently, trans-sacral, epiduroscopicassisted, 1,414-nm Nd:YAG laser decompression (SELD) is commonly used for spinal pathologies and has been used as a minimally invasive procedure because it is associated with better visualization, precise pathology targeting, minimal collateral tissue disruption, and less bleeding. These advantages make the surgery easier to perform, improve healing, and minimize the risk of patient discomfort ${ }^{7,14,18)}$. To the best of our knowledge, however, no studies have assessed the efficacy of the SELD-assisted, 1,414-nm Nd:YAG laser for the treatment of lumbar discal cyst. The purpose of this study was to report nine cases of lumbar discal cyst that were treated with SELD-assisted, 1,414-nm $\mathrm{Nd}$ :YAG laser and discuss the relevant literature.

\section{CASES REPORT}

Nine consecutive patients were treated with a SELD-assisted, 1,414-nm Nd:YAG laser for discal cyst at four institutions from 2014 to 2015. We obtained IRB approval for the study and written informed consent from patients. We then examined nine patients with low back pain and leg pain caused by a discal cyst. All 
patients were treated with a SELD-assisted, 1,414-nm Nd:YAG laser and approximately one year follow up. Epiduroscopy was performed under fluoroscopy in a sterile operating room with monitoring equipment for blood pressure, pulse rate, and pulse oximetry. Fluoroscopy was adjusted over the lumbosacral area such that a caudal approach could be used for both the anteroposterior and lateral views. After appropriate positioning of the fluoroscope, the needle insertion area was determined around the sacral hiatus and was injected with local anesthetics.

A 5-mm skin incision was made over the sacral hiatus using a number 11 blade. The sacrococcygeal ligament was punctured with a Tuohy needle under fluoroscopic guidance, and a Myelotec catheter was inserted into the sacrococcygeal ventral epidural space. Subsequently, a 3.0-mm steerable VGC was introduced, and the catheter was advanced into the lumbar epidural space under C-arm anterior/posterior (AP) and lateral views. An epidurogram was performed by using approximately $5 \mathrm{~mL}$ of a non-iodinated contrast agent (IOBRIX, ACCUZEN, Seoul, Korea). Identification of filling defects was attained by examining the contrast agent flow. We confirmed that there was no intravascular or subarachnoid placement of the needle; if such malpositioning occurred, the needle was repositioned. The Nd:YAG laser (Lutronics, Ilsan, South Korea) was introduced through the epiduroscope (MYELOTEC, Roswell, Georgia, USA), and the discal cyst was identified between the posterior longitudinal ligament (PLL) and the posterior annulus. Once identified, the cyst was ablated (Fig. 1). The intravenous line and epidural catheter were removed, and the patient was discharged if clinical
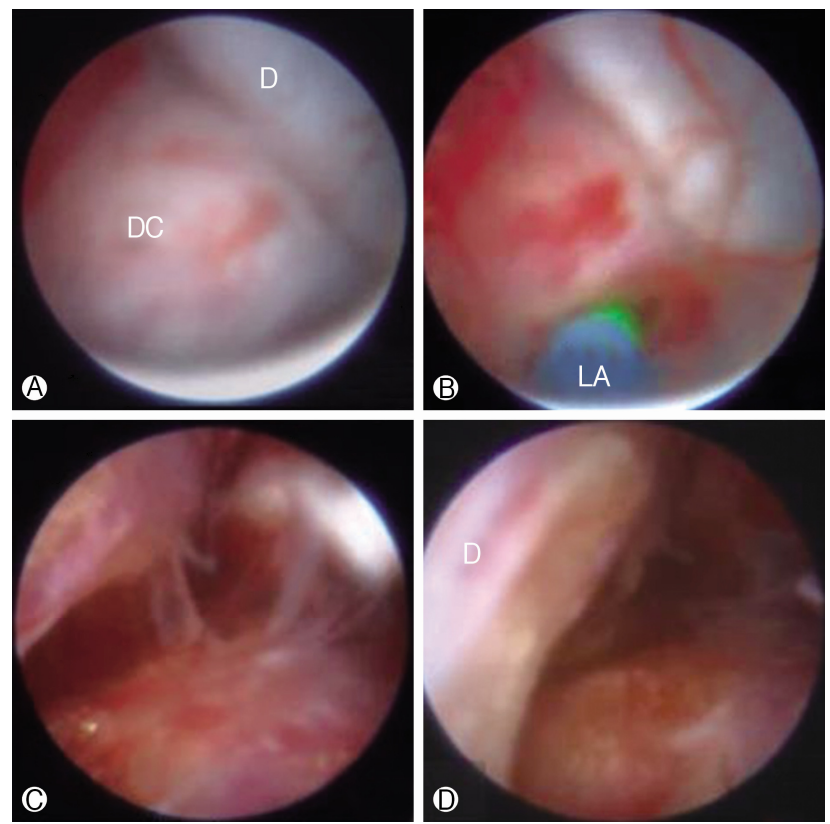

Fig. 1. Epiduroscopic-assisted laser view. (A) Duramater compressed by discal cyst. (B) and (C) the discal cyst was decompressed by the Nd:YAG laser (Luxton; BioVision; Gyeonggido, Korea). (D) after ablation freed the nerve root ( $D$, dura mater; DC, discal cyst; LA=LASER ABLATION). symptom were improved. The first follow-up was performed one week after the procedure. During one week follow-up, all patients received nonsteroidal anti-inflammatory drugs (NSAIDs) and muscle relaxants of equal doses to reduce procedure-related pain ${ }^{4,16)}$.

\section{Statistical Analysis}

The paired t-test was used to compare VAS in the pre and post procedures. Statistical analysis was performed using SPSS software package version 10 (SPSS Inc., Chicago, IL, USA), and all values were expressed as mean \pm standard deviation (SD) and defined as statistically significant in the case of $p<0.05$.

\section{1) Case 1}

A 20-year-old man was admitted to our hospital due to back pain that had lasted for 8 months, but the symptoms were relieved if the patient rest. He received intermittent physiotherapy, acupuncture, and other symptomatic treatment for a while. One month before admission, the patient began to feel severe back pain Preoperative physical examination revealed a positive straight leg raising test for the left leg $\left(70^{\circ}\right)$. The right lower limb had normal muscle force and knee reflex, and the bilateral Achilles tendon reflexes were normal.

Bilateral pathological reflex was not elicited. The preoperative visual analog scale (VAS) score for back pain was 8 (Fig. 2).
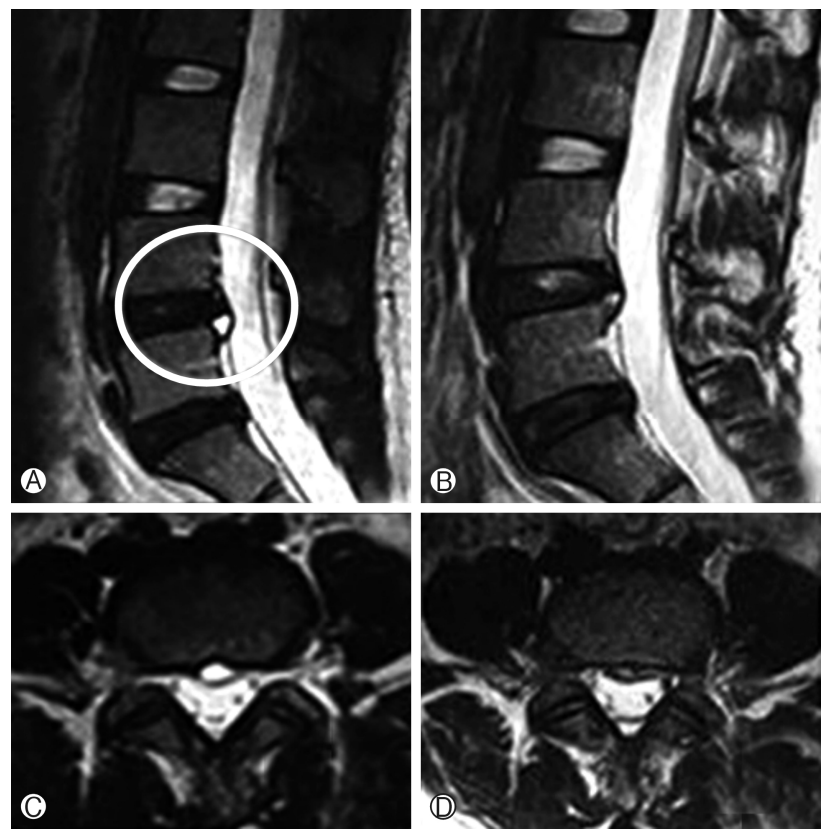

Fig. 2. (A, (B) Hyperintense on MRI T2-weighted image sagittal view showing a interior discal cyst before and after ablation. (C), (D) T2-weighted image axial view and surgically confirmed to be a discal cyst. 


\section{2) Case 2}

A 31-year-old man was admitted to our hospital due to left leg radiating pain that had lasted for one year. Before admission, he had received physical rehabilitation treatment. Three months before admission, the patient began to feel radiating pain in the left lower limb with numbness, mainly in the front thigh and medial calf, which got worse after activity. Preoperative physical examination revealed a positive straight leg raising test for the left leg $\left(30^{\circ}\right)$. The right lower limb had normal muscle force. The left knee reflex was reduced, but the right knee reflex and bilateral Achilles tendon reflexes were normal. Bilateral pathological reflex was not elicited. The preoperative VAS score was 4 points for back pain and 7 points for pain in the left leg (Fig. 3).

\section{3) Case 3}

A 30-year-old woman was admitted to our hospital due to left leg radiating pain and chronic low back pain that had lasted for one year and was aggravated by sitting and walking. The patient underwent treatment with anti-inflammatory medication and muscle relaxants. One week before admission, the patient began to feel severe pain in the left leg. Preoperative physical examination revealed a positive left straight leg raising test $\left(30^{\circ}\right)$. The right lower leg had normal muscle force, and the bilateral knee reflex was normal. The bilateral pathological reflex was also normal. The preoperative VAS for back pain was 8 points (Fig. 4).

\section{RESULTS}

All of the patients suffered from unilateral lower extremity pain and back pain. Although the straight leg raising test was positive in seven patients, chronic low-back pain was present in only one of these patients. Preoperative MRI showed the cyst connected to the intervertebral disc. All MR images of patients showed low signal intensity in T1-weighted images and high signal intensity in T2-weighted images and a round to oval mass lesion on MRI. These findings are compatible with a liquid-containing cyst. Back pain and radiating pain were improved in all patients at the final follow-up. The mean VAS score for pain was $7.89 \pm 0.78$ preoperatively, $1.67 \pm 1.50$ at onemonth follow-up, and $0.38 \pm 0.5$ at the final follow-up $(p<0.01)$ (Table 1).

According to MacNab's criteria, two patients (22.2\%) had excellent results and 7 patients (77.7\%) had good results. No patients had any complications. There was no evidence of cyst recurrence during follow-up.

\section{DISCUSSION}

Lumbar discal cysts are rare and are therefore an uncommon cause of lumbar radiculopathy. They usually occur in the third or fourth decade of life ${ }^{2,10}$. Previous studies have found that the mean age of patients with lumbar discal cyst is $30.78 \pm 4.84$ years (range, 20 to 39 years), younger than the population with lumbar herniated disc. Discal cysts may form due to mechanical stress-induced focal degeneration of the adjacent intervertebral disc wall, annulus fibrosis, and fluid collection, followed by the formation of a pseudomembrane around the fluid collection ${ }^{1,5,10)}$.
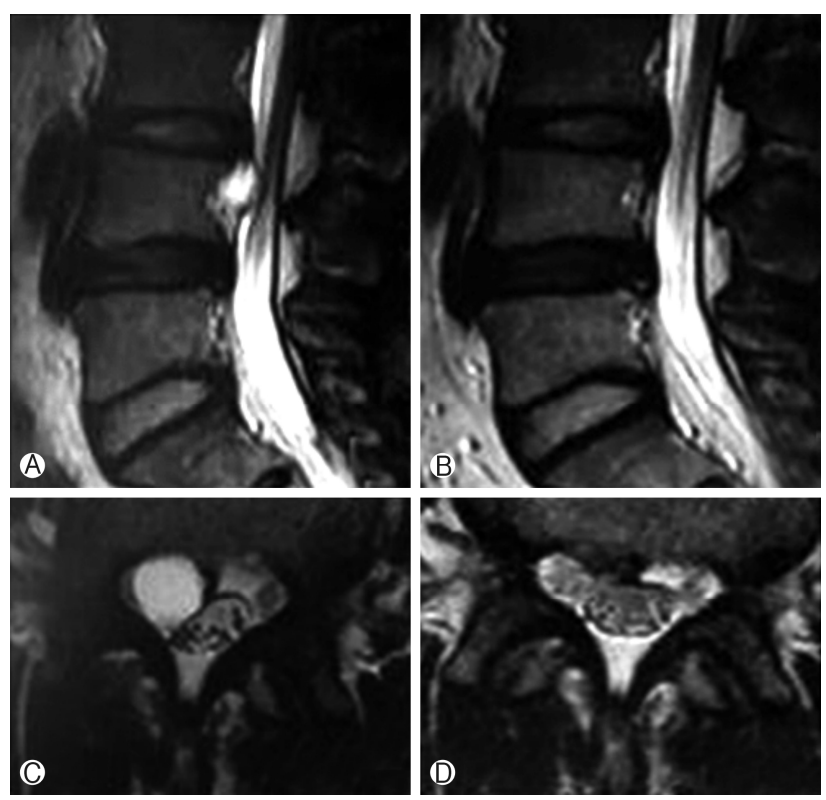

Fig. 3. (A), B) MRI T2-weighted image sagittal view showing a left-sided oval-shaped cyst originating from the left $L 4-5$ disc before and after ablation. (C, (D) MRI T2-weighted image axial view showed discal cyst on the left side before and after ablation.
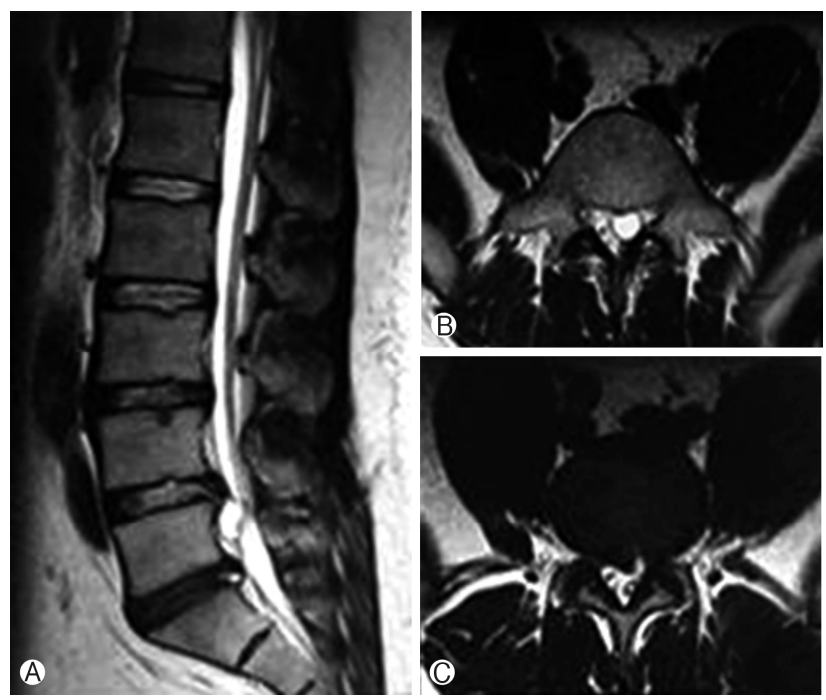

Fig. 4. (A) MRI hyperintense on T2-weighted image sagittal view revealed discal cyst before ablation, (B) T2-weighted image axial view showed a discal cyst in the left side, (C) MRI T2-weighted image axial view after ablation. 
Table 1. Demographics of patients with Discal Cysts

\begin{tabular}{|c|c|c|c|c|c|c|c|c|}
\hline \multirow{2}{*}{ Sex } & \multirow{2}{*}{ Age (yrs) } & \multirow{2}{*}{ Level } & \multicolumn{4}{|c|}{ VAS } & \multirow{2}{*}{ MacNabs } & \multirow{2}{*}{ Complication } \\
\hline & & & Pre op & $1 \mathrm{mo}$ & $6 \mathrm{mo}$ & $1 \mathrm{yr}$ & & \\
\hline$F$ & 30 & $\llcorner 4-4$ & 8 & 4 & 1 & 0 & Good & No \\
\hline$M$ & 31 & $\llcorner 4-5$ & 7 & 0 & 0 & 0 & Excellent & No \\
\hline $\mathrm{M}$ & 32 & L3-4 & 8 & 0 & 0 & 0 & Excellent & No \\
\hline $\mathrm{M}$ & 32 & L5-S1 & 9 & 0 & 0 & 1 & Good & No \\
\hline $\mathrm{M}$ & 31 & L3-4 & 7 & 1 & 1 & 1 & Good & No \\
\hline$F$ & 39 & L3-4 & 9 & 2 & 1 & 1 & Good & No \\
\hline M & 20 & $\llcorner 4-5$ & 8 & 3 & 1 & 0 & Good & No \\
\hline $\mathrm{M}$ & 31 & L3-4 & 7 & 2 & 1 & 0 & Good & No \\
\hline $\mathrm{F}$ & 30 & $\llcorner 4-5$ & 8 & 3 & 1 & 0 & Good & No \\
\hline
\end{tabular}

VAS: Visual Analogue Scale; ${ }^{\S}$ Macnabs: Outcome assesment of patients satisfaction

This study included six male patients (66.6\%) and three female patients (33.4\%). The higher prevalence of discal cyst in males may result from greater shear stress due to greater physical activity and/or hormonal influences in males compared to females. All nine patients in this study were Asian, while the majority of patients in a similar study by Aydin et al were Japanese and South Korean ${ }^{1,5,10)}$. Currently, most discal cysts are managed by partial hemi laminectomy and microscopic resection of the cyst under general anesthesia. CT-guided aspiration is another minimally invasive option, but it is associated with a high recurrence rate and requires radical excision of the intraspinal cyst ${ }^{6,10,19)}$.

Other reports have completed resection by percutaneous endoscopic surgery, but the recurrence rate with percutaneous $\mathrm{C}$-arm guide aspiration is not yet known. Recurrence has been reported by Lee et al and Kang et al. after surgical removal of a discal cyst, but no studies have assessed recurrence after treatment with the 1,414-nm Nd:YAG laser ${ }^{1,5,6,9-11,17,19,20)}$.

As the most commonly used laser for ablation therapy, the Ho:YAG laser has been accepted as the safest laser because it has low tissue penetration. Although the $\mathrm{Nd}$ :YAG laser is not considered appropriate for deep tissue penetration, it can provide shallow tissue penetration by adjusting its wavelength to that of the Ho:YAG laser. Easier laser control, lower manufacturing cost, and similar effectiveness are other advantages of the $\mathrm{Nd}: \mathrm{YAG}^{3,4,8,13,14)}$.

An in vivo study reported that 6-Watt delivery of the 1,414-nm $\mathrm{Nd}$ :YAG laser and the addition of an epiduroscope led to lower heat delivered to the surrounding ablated tissue, deeper tissue penetration, and increased target accuracy. This reduced the risk of thermal injury and made the 1,414-nm Nd:YAG laser safe for use in humans ${ }^{12,14)}$.

Our study confirmed that a SELD-assisted, 1,414- nm Nd:YAG laser can effectively treat lumbar discal cyst ${ }^{7,12,14)}$. However, this study had some limitations. The sample size was small, so double-blind, controlled studies with a higher number of patients are needed to confirm efficacy. In addition, we did not assess quality of life through the SF-36 or a similar instrument. Nevertheless, this study is valuable because it highlights the association between disca cyst ablation with the endoscopic-assisted, 1,414$\mathrm{nm} \mathrm{Nd}$ :YAG laser and clinical outcomes.

In these cases, no residual cysts appeared after surgical treatment or on postoperative MRI. There were no neurologic deficits and no signs of local recurrence on MRI at the 12-month follow-up, but regular follow-up evaluations are required.

Standard therapeutic guidelines for discal cysts have not been established because the natural history and long-term prognosis of discal cysts treated by surgery or percutaneous CT-guided aspiration and steroid injection are still unknown. However, we believe that the operative indications for discal cyst are likely to be similar to those for lumbar disc herniation. In a current study, discal cysts were treated effectively by a SELD-assisted, 1,414-nm Nd:YAG laser with preservation of the disc, even though it is generally believed that a discal cyst may recur when the cyst is only partially removed. To provide more definitive data on the natural course and standard treatment for discal cysts, more studies of discal cyst treatment accompanied by careful analysis and long-term follow-up are necessary.

\section{CONCLUSION}

Trans-sacral, epiduroscopic-assisted, 1,414-nm Nd:YAG laser decompression was a safe, viable, and efficacious option for lumbar discal cyst treatment because it reduced the risk of muscle and thermal injury and can be performed under local anesthesia.

\section{ACKNOWLEDGEMENT}

This study was supported by a grant from the Korea Health Technology R\&D Project through the Korea Health Industry Development Institute (KHIDI), funded by the Ministry of Health and Welfare, Republic of Korea (HC15C1320). 


\section{Disclaimer}

The authors received no external funding to prepare this manuscript.

\section{Conflict of Interest}

Each author certifies that he or she, or a member of his or her immediate family, has no commercial association (i.e., consultancies, stock ownership, equity interest, patent/licensing arrangements, etc.) that might pose a conflict of interest in connection with the submitted manuscript.

\section{REFERENCES}

1. Aydin S, Abuzayed B, Yildirim H, Bozkus H, Vural M: Discal cysts of the lumbar spine: report of five cases and review of the literature. Eur Spine J 19:1621-1626, 2010

2. Certo F, Visocchi M, Borderi A, Pennisi C, Albanese V, Barbagallo GMV: Lumbar intervertebral discal cyst: a rare cause of low back pain and radiculopathy. case report and review of the current evidences on diagnosis and management. Evid Based Spine Care J 5:141-148, 2014

3. Choy DS, Altman P, Trokel SL: Efficiency of disc ablation with lasers of various wavelengths. Journal of Clinical Laser Medicine \& Surgery 13:153-156, 1995

4. Choi G, Uniyal P, Kim WH, Hassan Z, Patel B Lee JH: Epiduroscopic assisted percutaneous endoscopic lumbar discectomy: a technical report. J Neurol Disord 4:2-5, 2016

5. Ha SW, Kim SW, Kim YH, Kim HS: Clinical outcomes of percutaneous endoscopic surgery for lumbar discal cyst. J Korean Neurosurg Soc 51:208-214, 2012

6. Jha SC, Sakai T, Takata Y, Abe M, Nagamachi A, Fukuta S, Sairyo K: Percutaneous endoscopic discectomy via transforaminal route for discal cyst. Case Reports in Orthopedics:1-5, 2015

7. Johnson MR, Hill WM, Boettcher T: Ablation of porcine ligamentum flavum with Ho:YAG, q-switched Ho:YAG and quadrupled Nd:YAG lasers. Lasers in Surgery and Medicine 47:839851,2015

8. Jayasree R, Gupta AK, Bodhey NK, Mohanty M: Effect of 980$\mathrm{nm}$ diode laser and 1,064-nm Nd:YAG laser on the intervertebral disc in vitro and in vivo studies. Photomedicine and Laser
Surgery 27:547-552, 2009

9. Kang H, Liu WC, Lee SH, Paeng SS: Midterm results of percutaneous CT-guided aspiration of symptomatic lumbar discal cysts. Am J Roentgenol 190:310-314, 2008

10. Kang H, Lee SH, Paeng SS: Midterm results of percutaneous ct-guided aspiration of symptomatic lumbar discal cysts. AJR 190:W310-W4, 2008

11. Lee HK, Lee DH, Choi CG, Kim SJ, Suh DC, Kang SK, et al: Discal cyst of the lumbar spine: MR imaging features. Clin Imaging 30:326-330, 2006

12. Lee SH, Lee SH, and Lim KT: Trans-sacral epiduroscopic laser decompression for symptomatic lumbar disc herniation: a preliminary case series. Photomedicine and Laser Surgery 34:121129, 2016

13. Marguet CG, Sung JC, Springhart WP, L'esperance JO, Zhou S, Zhong P, Albala DM, Preminger GM: In vitro comparison of stone retropulsion and fragmentation of the frequency doubled, double pulse nd:yag laser and the holmium:yag laser. The Journal of Urology 173:1797-1800, 2005

14. Moon BJ,Yi S, Ha Y, Yoon DH, Kim KN, Shin DA: Experimental evaluation of percutaneous lumbar laser disc decompression using a 1,414 nm Nd:YAG Laser. Pain Physician 18:E1091E1099, 2015

15. Murata K, Tanaka C, Kanoe H, Okudaira S: Discal cysts of the lumbar spine: a case report. Journal of Orthopaedic Surgery 15: 376-379, 2007

16. Oh CH, Ji GY, Cho PG, Choi WS, Shin DA, Kim KN, Kang HA: The catheter tip position and effects of percutaneous epidural neuroplasty in patients with lumbar disc disease during 6-months of follow-up. Pain Physician 17:E599-E608, 2014

17. Park JM, Lee GW: The effectiveness of transforaminal epidural injection for discal cyst of lumbar spine. J Minim Invasive Orthop 1:1-8, 2014

18. Singh V, Machikanti L, Calodney AK, Staats PS, Falco FJE, Caraway DL, Hirsch JA, Cohen SP: Percutaneous lumbar laser disc decompression: an update of current evidence. Pain Physician 16:SE229-SE260, 2013

19. Wang ES, Kim SW, Kim YS, Kim DM: Clinical analysis of microscopic removal of discal cyst. Korean J Spine 10:61-64, 2013

20. Yu HJ, Yim KH: Successful treatment of a symptomatic discal cyst by percutaneous c-arm guided aspiration. Korean J Pain 29:129-135, 2016 